\title{
GESTIÓN EMPRESARIAL DE LA INDUSTRIA CEMENTERA PERUANA, LA CORRUPCIÓN Y EL NIÑO COSTERO, 2016-2017
}

\author{
BUSINESS MANAGEMENT OF THE PERUVIAN CEMENT INDUSTRY, \\ CORRUPTION AND THE COASTAL CHILD, 2016-2017
}

Raúl Alberto Arrarte Mera Universidad Nacional Mayor de San Marcos Lima, Perú ORCID: http://orcid.org/0000-0002-7222-7752 Correo electrónico: rarrartem@unmsm.edu.pe

Emilio Gabriel García Villegas Universidad Nacional Mayor de San Marcos Lima, Perú ORCID: http://orcid.org/0000-0002-5099-7342 Correo electrónico: egarciav@unmsm.edu.pe

\section{RESUMEN}

Objetivo: Analizar si la gestión empresarial de la industria cementera peruana superó los problemas de la corrupción y el Niño Costero en el periodo 2016-2017. Método: Es una investigación de tipo aplicada, documental y descriptiva, con diseño no experimental que pretende dar razones del porqué de los fenómenos en estudio. La muestra es de un censo del sector cemento que abastece a todo el Perú. Resultados: El análisis estructural de las inversiones nos muestra una empresa típicamente industrial que prioriza el largo plazo en vez del corto; $85 \%$ de los activos no corrientes en el 2017 y 88\% en el año 2016. Conclusiones: La rentabilidad patrimonial en la industria cementera fue de más a menos en el periodo 2016 al 2017, excepto la empresa Cementos Yura, que tuvo rendimiento superior al $10 \%$ en ambos periodos de 2016 y 2017. En cuanto al valor económico agregado, todas las empresas tuvieron saldos negativos y no crearon valor para los accionistas en el periodo bajo estudio.

Palabras clave: Industria; gestión; corrupción; fenómeno; costa.
[Recibido: 11/10/2018 Aceptado: 02/08/2019 Publicado: 28/08/2019]

\begin{abstract}
Objective: Analyze if Peruvian cement industry business management overcame the problems of corruption and the Niño Costero in the 2016-2017 period. Method: It is a applied, documentary and descriptive research type, with non - experimental design that aims to give explain the reasons for the phenomena under study. Results: The sample is from a census of the cement sector that supplies all Peru, the structural investments analysis shows us a typical industrial company that prioritizes long term instead of short term; 85\% in 2017 and $88 \%$ in 2016 of non-current assets. Conclusions: The equity return in the sector went from more to less in the 2016 to 2017 period, except for Cementos Yura, which outperformed more than $10 \%$ in both periods (2016 and 2017). Regarding the added economic value, all the companies had negative balances and did not create value for the shareholders.
\end{abstract}

Keywords: Industry; management; corruption; phenomenon; coast

(c) Los autores. Este artículo es publicado por la Revista Quipukamayoc, Universidad Nacional Mayor de San Marcos. Este es un artículo de acceso abierto, distribuido bajo los términos de la Licencia Creative Commons Atribución-NoComercial-Compartirlgual 4.0 Internacional.(http://creativecommons.org/licenses/by-nc-sa/4.0/), que permite el uso no comercial, distribución y reproducción en cualquier medio, siempre que la obra original sea debidamente citadas. 


\section{INTRODUCCIÓN}

Los escándalos de corrupción revelada en el Perú los últimos meses del año 2018, vía el denominado caso Lava Jato, asociado a una firma constructora brasileña, mostraron el modo de operación negativo de sus pares peruanos. El problema es que son principales contribuyentes las involucradas, por tanto, tienen encadenamientos importantes hacia adelante y hacia atrás, provocando que la paralización de las mismas tenga un efecto multiplicador muy grande en la economía nacional porque sus proveedores y clientes se verán afectados en la cadena de cobros-pagos, lo que generaría un riesgo sistémico en las finanzas públicas. Si se detiene la construcción, principal motor de crecimiento del año 2017, no se generará decenas de miles de puestos de trabajo. Importantes empresas de este sector, cerca de 40 que han sido involucradas en el llamado club de la construcción, enfrentan serios problemas para recibir financiamiento bancario y están a la espera de un consenso sobre la norma que reemplazará al Decreto de Urgencia 003 para garantizar la continuidad de las obras de infraestructura.

Por otro lado, el desempeño de la economía en su conjunto se muestra así en el 2017, según el Instituto Nacional de Estadística e Informática (INEI, 2018):

En el cuarto trimestre del año 2017, el Producto Bruto Interno (PBI) a precios constantes de 2007, registró un crecimiento de $2,2 \%$, explicado por la evolución favorable de la demanda interna (5,0\%), en un contexto de crecimiento de las principales economías desarrolladas, con repercusiones positivas en los precios de las materias primas en particular de los metales.

En el crecimiento del PBI (2,2\%), incidió el incremento del consumo final privado en 2,6\%, consumo del gobierno (11,5\%) y del buen desempeño de la inversión privada $(6,2 \%)$ y pública $(4,3 \%)$. Esta sincronía del gasto privado y público se reflejó en el crecimiento de la demanda interna en 5,0\%, tasa mayor a las registradas desde el tercer trimestre del 2013 (p.1)

Aunque el PBI, disminuyó con referencia al año 2016, es importante hacer notar, desde el punto de vista del tema que analizamos, la inversión pública en infraestructura se recuperó a 4,3\% contra $-14,6 \%$ de 2016 . Se habría recuperado del impacto del Fenómeno del Niño Costero (FEN), y los juicios judiciales que se iniciaron por coincidencia en ese mismo año.

Pero volvamos a la información oficial del año 2016, para conocer qué sucedió con el PBI ese mismo año. Según INEI (2017) se señala que:
En el cuarto trimestre del año 2016, el PBI a precios constantes de 2007, registró un crecimiento de 3,0\% impulsado por el consumo privado en un contexto de recuperación de la economía mundial, con un incremento en las cotizaciones internacionales de nuestros principales productos de exportación; así como, por la implementación de medidas para impulsar el crecimiento económico. La inversión pública disminuyó en -14,6\% por la reducción de obras de infraestructura en los gobiernos nacional y regional $y$ en menor medida en los gobiernos locales; la inversión privada se contrajo en $-3,5 \%$ explicado por la disminución en la inversión minera, inversión en hidrocarburos, entre otras (p.2).

El PBI se vio afectado principalmente por la disminución de la inversión pública, mostrando los primeros impactos cuantificados del FEN. Veamos lo que nos dicen Contreras, Martínez, Regalado y Vásquez (2017), quienes al respecto señalan:

El Fenómeno de El Niño (FEN), es un evento natural oceánico-atmosférico que se caracteriza por un calentamiento intenso y anormal de las aguas superficiales del mar en el Océano Pacífico Ecuatorial frente a las costas de Perú y Ecuador, generando cambios climáticos a nivel regional y global (p.3).

Entre enero y mayo de 2017, caracterizado como extraordinario, generó intensas lluvias en zonas desérticas de la costa, afectando ciudades, vías de comunicación y valles productivos que se vieron inundados de agua, afectando la vida de las regiones principalmente de Trujillo, Chiclayo, Piura y Tumbes.

Frente a esta realidad nos preguntamos, ¿la gestión empresarial de la industria cementera peruana superó los problemas de la corrupción y el FEN en el periodo 2016-2017?

Según Arrarte (2012):

Aplicando este análisis pretendemos demostrar, cuán importante es conocer la evolución de la estructura de inversión de corto y de largo plazo; y la estructura de financiamiento en capital propio y ajeno, para señalar el estilo de cómo financia sus operaciones el sector bajo estudio (p.201).

Un aspecto fundamental en el financiamiento de las operaciones, es la forma cómo se prioriza el capital propio respecto del capital ajeno. Pensando en el criterio que, a más endeudamiento, altos intereses a pagar y a menor endeudamiento, más dividendos por abonar a los accionistas. Finalmente, el crecimiento del capital propio siempre debe ser el objetivo más significativo que el del capital ajeno. 
Otro evento importante a superar en el mercado interno, es el efecto económico-social del FEN, que, según Contreras et al (2017) lo expresan así:

El FEN es un evento océano-atmosférico donde aguas cálidas sub-superficiales se trasladan a lo largo del Pacífico Tropical y se produce un debilitamiento sostenido de los vientos alisios. Estos factores incrementan la temperatura superficial del mar y alteran el comportamiento de otras variables climatológicas. Entre los principales efectos y manifestaciones de El Niño destacan la menor cantidad anual de huracanes en el Atlántico, inviernos cálidos o alteraciones del ciclo hidrológico durante el verano de América del Sur (Perú, Ecuador y Chile), sequías e incendios forestales en Oceanía y cambios en el ciclo hidrológico desde marzo a mayo en África (p.3).

Para entender el impacto negativo de este fenómeno, acudamos nuevamente a la opinión autorizada de Contreras et al. (2017) quienes afirman:

Respecto a la actividad económica el FEN representa un típico caso de shock de oferta negativo, generando presiones al alza en los precios y efectos contractivos sobre la actividad productiva. Cabe precisar que este fenómeno afecta directamente a aquellas actividades que dependen de factores climáticos debido a que las anomalías térmicas y/o pluviales generan efectos directos sobre la producción de bienes, o influyen indirectamente a través de daños a la infraestructura que impiden el normal desarrollo de otras actividades como el comercio, transporte, y otros servicios.

En el caso de Perú los efectos del FEN generan efectos en la actividad económica desde que comienza la alerta de este fenómeno, dado en su impacto en las expectativas, lo cual afecta a las intenciones de siembras, al crédito a los productos agrícolas vulnerables e incrementa la demanda de productos relacionados a la prevención o a mitigar su impacto (seguros contra desastres naturales y otros). Asimismo, el FEN genera oscilaciones en los precios agrícolas (por menor abastecimiento), en la balanza comercial por menores exportaciones y mayores importaciones y en la recaudación por los desbalances antes mencionados (p.3).

Según Weston (1995), “el análisis financiero tradicional se ha centrado en las cifras. El valor de este enfoque radica en que se pueden utilizar ciertas relaciones cuantitativas para diagnosticar los aspectos fuertes y débiles del desempeño de una empresa" (p.215).

Para Arrarte (2012):

Alfred Marshall fue el primero que expresó una noción de EVA, en 1980, en su obra capital The Prin- ciples of Economic: Cuando un hombre se encuentra comprometido con un negocio, sus ganancias para el año son el exceso de ingresos que recibió del negocio durante al año sobre sus desembolsos en el negocio. La metodología de EVA supone que el éxito empresarial está relacionado directamente con la generación de valor económico, que se calcula restando a las utilidades operacionales el costo financiero por poseer los activos que se utilizaron en la generación de dichas utilidades (p.202).

Analizar si la gestión empresarial de la industria cementera peruana superó los problemas de la corrupción y el FEN en el periodo 2016-2017 es uno de los objetivos de la investigación.

\section{MATERIAL Y MÉTODOS}

El diseño utilizado en la investigación es no experimental porque no se actúa conscientemente sobre el objeto de estudio. Es Aplicada, porque busca la generación de conocimiento colaborando entre la universidad y la industria cementera en el proceso de transferencia de tecnología. Documental, porque procede de publicaciones de información financiera de la Bolsa de Valores de Lima (BVL), de tres empresas cementeras más importantes del Perú. Explicativa, porque pretende dar razones del porqué de los fenómenos en estudio. La muestra fue obtenida de un censo del sector cementos que abastece a todo el Perú, el norte, para Cementos Pacasmayo; el centro para UNACEM y el sur para Cementos Yura. Hacemos notar que los estados financieros utilizados en el desarrollo de este trabajo fueron al 31 de diciembre de 2016 y al 30 de setiembre de 2017.

A continuación, procedimos a determinar el costo de capital de cada una de las empresas cementeras bajo análisis, de acuerdo a los siguientes pasos:

a. Al Activo Corriente se restó el Pasivo Corriente para obtener el Capital Neto de Trabajo.

b. A este monto se agregó el Activo Neto para obtener el Total de Recursos.

c. Al Total de Recursos, finalmente, se aplicó una tasa estimada del 7\%, para lograr el costo de capital (ver tabla 7 para el 2016; Tabla 8, para el 2017).

El paso final es calcular el Valor Económico Agregado (VEA), según el procedimiento siguiente:

a. Identificamos la Utilidad Operativa Antes de Impuestos (UOAI) en la primera columna procedente de los Estados de Resultados. 
b. Deducimos el $27 \%$ de Impuestos a la Renta (IR), en la Segunda Columna.

c. Identificamos la Utilidad Neta Después de Impuestos (UNDI), a la diferencia de la UOAI, menos el IR.

d. A la UNDI, finalmente se deduce el Costo de Capital (CDC) ya calculado en las Tablas 10 y 11, y establecemos el VEA. (ver tabla 7 para el 2016; Tabla 8, para el 2017).

\section{RESULTADOS}

Una de las primeras explicaciones de por qué se expandió la corrupción en el periodo bajo análisis, y qué hizo metástasis, fue la ausencia de coordinación entre las instituciones tutelares del país: La Contraloría General de la República (CGR), el Ministerio Público (MP), el Poder Judicial (PJ) y la Unidad de Inteligencia Financiera (UIF).

El sector construcción representa algo más del 6\% del Producto Bruto Interno (PBI); y en nuestro país los últimos años ha sido uno de los más dinámicos, pero la desaceleración de la economía provocó una caída en el sector, aquel que genera empleos y tiene un efecto multiplicador muy grande en la economía.

Este flagelo económico-social que afecta a todos los peruanos, ocurrió en los gobiernos constitucionales de los expresidentes: Alejandro Toledo Manrique, Alan García Pérez y Ollanta Humala Tasso, contratos de infraestructura por más de 10987 millones de dólares americanos y adendas por más de 2282 millones de dólares adicionales como se detalla en la tabla 1.

La corrupción es un tema analizado por muchos, pero aún con pocos resultados prácticos (ver tabla 2 ).

Los exfuncionarios públicos que son actualmente investigados por corrupción, en la Fiscalía de la Nación, el Poder Judicial y la Contraloría General de la República peruana se presentan en la tabla 3.

La Contraloría General de la República controla y detecta la irregularidad; el Ministerio Público investiga y confirma las pruebas; el Poder Judicial aplica la ley y la Unidad de Inteligencia Financiera traza el itinerario del dinero mal habido; así se demostraría la existencia de una política de estado contra la corrupción.

Para optimizar el funcionamiento de la Contraloría, se propone:

a. Reestructurar su Organigrama en cinco regiones descentralizadas.

b. El Personal deberá rotarse obligatoriamente al término del tercer año calendario.

c. La estructura de especialización deberá adaptarse a los siguientes procesos:

- Control Previo.

- Control Posterior.

- Informe Final.

Tabla 1

Proyectos con firmas brasileñas con mayor incremento presupuestal. (Expresado en USD).

\begin{tabular}{|c|c|c|c|c|c|}
\hline No. & Proyecto & Empresa Investigada & $\begin{array}{l}\text { Presupuesto } \\
\text { Inicial }\end{array}$ & $\begin{array}{l}\text { Incremento } \\
\text { en Costos }\end{array}$ & Año \\
\hline 1 & Olmos Trasvase & $\begin{array}{l}\text { Odebrecht Perú Inversiones en Infraestructura } \\
\text { S.A. - Constructora Norberto Odebrecht S.A. }\end{array}$ & 185000000 & 96000000 & 2004 \\
\hline 2 & IIRSA Sur Tramo 4 & $\begin{array}{l}\text { Constructora Andrade Gutiérrez- Construcoes } \\
\text { e Comercio Camargo Correa S.A. - Construc- } \\
\text { tora Queiroz Galvao S.A. }\end{array}$ & 244000000 & 437000000 & 2005 \\
\hline 3 & IIRSA Norte Paita-Yurimaguas & Andrade Gutiérrez S. A. & 258000000 & 290000000 & 2005 \\
\hline 4 & IIRSA Sur, Tramo 3 & Constructora Norberto Odebrecht S.A. & 395000000 & 230000000 & 2005 \\
\hline 5 & IIRSA Sur Tramo 2 & Constructora Norberto Odebrecht S.A. & 263000000 & 396000000 & 2006 \\
\hline 6 & Metro de Lima 1.Tramo 1 & Constructora Norberto Odebrecht S.A. & 410000000 & 109000000 & 2009 \\
\hline 7 & Metro de Lima 1.Tramo 2 & Constructora Norberto Odebrecht S.A. & 583000000 & 302000000 & 2011 \\
\hline 8 & Central Hidroeléctrica Chaglla & Constructora Norberto Odebrecht S. A. & 1247000000 & 224000000 & 2011 \\
\hline 9 & Hospital Antonio Lorena Nivel 3 & Constructora OAS Ltda. Sucursal Perú & 74000000 & 62121212 & 2013 \\
\hline 10 & Gasoducto Sur Peruano & Odebrecht Latinvest Perú Kuntur S.A. & 7328000000 & 136000000 & 2014 \\
\hline TOTALES & & & $\begin{array}{r}10987000 \\
000\end{array}$ & 2282121212 & \\
\hline
\end{tabular}

Fuente: Comisión Lava Jato del Congreso Nacional. Lima. Perú. 
Tabla 2

Definiciones de corrupción.

\begin{tabular}{|c|c|c|c|}
\hline \multicolumn{3}{|c|}{ La corrupción } & Autores \\
\hline \multicolumn{3}{|c|}{$\begin{array}{l}\text { "No es esencialmente un problema cultural. Se trata, en cambio, de un problema institucional que, por } \\
\text { tanto, no puede ser efectivamente atacado solamente con el sistema de persecución penal". }\end{array}$} & $\begin{array}{c}\text { (Volosin, } \\
2015, \text { p. 8-9) }\end{array}$ \\
\hline \multicolumn{3}{|c|}{$\begin{array}{l}\text { "El Congreso peruano continúa funcionando como una legislatura unicameral bajo la actual y seria- } \\
\text { mente defectuosa Constitución de 1993. Los intereses corruptos siguen cabildeando en pos de la im- } \\
\text { punidad y reformas cosméticas que puedan ocultar las ganancias ilegales de unos cuantos escogidos" }\end{array}$} & $\begin{array}{l}\text { (Quiroz, } \\
\text { 2013, p. 530) }\end{array}$ \\
\hline \multicolumn{4}{|c|}{$\begin{array}{l}\text { - El } 71 \% \text { de los encuestados considera que el peruano es poco honesto o nada honesto. } \\
\text { - Las instituciones peor evaluadas son: El Congreso (el } 79 \% \text { considera que es muy corrupto); el } \\
\text { Poder Judicial ( } 73 \% \text { lo considera muy corrupto); y el Gobierno Central ( } 71 \% \text { lo considera muy } \\
\text { corrupto). } \\
\text { - El } 86 \% \text { califica como poco o nada eficiente el desempeño del Poder Ejecutivo en su lucha contra } \\
\text { la corrupción. } \\
\text { - El } 50 \% \text { considera al gobierno de Alan García tan corrupto como los anteriores y } 20 \% \text { incluso más } \\
\text { que los anteriores. } \\
\text { - El } 58 \% \text { considera que la corrupción en el país será igual, algo peor o mucho peor en el futuro } \\
\text { inmediato. }\end{array}$} \\
\hline \multicolumn{3}{|c|}{$\begin{array}{l}\text { Es el mal uso de los poderes públicos, cargo o autoridad para beneficio propio mediante el soborno, } \\
\text { la extorsión, el tráfico de influencias, el nepotismo, el fraude, la extracción de dinero para agilizar trá- } \\
\text { mites, o la malversación de fondos. Si bien a menudo la corrupción se considera un problema de los } \\
\text { empleados públicos, también prevalece en el sector privado. }\end{array}$} & $\begin{array}{l}\text { Programa de Desarrollo de } \\
\text { las Naciones Unidas (PNUD) } \\
\qquad(2003) \text {. } \\
\text { USAID, }(2004, \text { p.15) }\end{array}$ \\
\hline \multicolumn{3}{|c|}{$\begin{array}{l}\text { Es el uso indebido del poder otorgado para beneficio privado. La corrupción entraña conductas por } \\
\text { parte de funcionarios en el sector público o sus allegados, por las cuales se enriquecen indebida e } \\
\text { ilegalmente mediante el mal uso del poder que se les ha confiado. }\end{array}$} & $\begin{array}{l}\text { Transparencia Internacional (TI) } \\
\qquad(2000) \\
\text { USAID, }(2004, \text { p.15) }\end{array}$ \\
\hline \multicolumn{3}{|c|}{$\begin{array}{l}\text { "Se define como el abuso de un puesto público para ganancia privada. El Banco Mundial desglosa el } \\
\text { concepto, y hace principal hincapié en la distinción entre la corrupción como captura del Estado y la } \\
\text { corrupción administrativa". }\end{array}$} & $\begin{array}{l}\text { Banco Mundial (BM) (2000) } \\
\quad \text { USAID, (2004,p.15) }\end{array}$ \\
\hline \multicolumn{3}{|c|}{$\begin{array}{l}\text { Es el abuso de un puesto público en beneficio privado. Abarca abusos unilaterales por funcionarios } \\
\text { públicos del gobierno como malversación de fondos y nepotismo, así como el abuso que vincula tanto } \\
\text { al sector público como al sector privado, tales como soborno, extorsión, tráfico de influencias y fraude. }\end{array}$} & $\begin{array}{l}\text { Agencia de los Estados Unidos } \\
\text { para el Desarrollo Internacional. } \\
\text { (USAID) (1999) }(2004, \text { p.15) }\end{array}$ \\
\hline \multicolumn{4}{|c|}{ Fuente: Tomado de USAID (2004, p.15). } \\
\hline \multirow{2}{*}{\multicolumn{4}{|c|}{$\begin{array}{l}\text { Tabla } 3 \\
\text { Cuadro de involucrados de acuerdo a la Fiscalía. }\end{array}$}} \\
\hline & & & \\
\hline Acusados de ser Testaferros & Nivel Ejecutivo & \multicolumn{2}{|l|}{ Cargo } \\
\hline Hernán Garrido Lecca & Primero & \multicolumn{2}{|l|}{ Exministro de Vivienda. } \\
\hline José Antonio Chang & Primero & \multicolumn{2}{|l|}{ Exministro de Educación } \\
\hline Aurelio Pastor Valdivieso & Primero & \multicolumn{2}{|l|}{ Exministro de Justicia } \\
\hline Luis Nava Guibert & Primero & \multicolumn{2}{|l|}{ Exsecretario de Palacio } \\
\hline Pilar Nores Bodereau & Primero & \multicolumn{2}{|l|}{ Ex primera dama } \\
\hline Asabedo Fernández Carretero & Segundo & \multicolumn{2}{|l|}{ Exsecretario general del Ministerio de Educación. } \\
\hline María Gamarra de Fernández & Segundo & \multicolumn{2}{|l|}{ Exfuncionaria de COFOPRI } \\
\hline Pedro Morales Gonzáles & Segundo & \multicolumn{2}{|c|}{ Exjefe de la Oficina de Infraestructura Educativa del Ministerio de Educación. } \\
\hline Luis Huaylinos Maraví & Segundo & \multicolumn{2}{|c|}{ Exjefe de la Oficina de Infraestructura Educativa del Ministerio de Educación. } \\
\hline Carlos Arana Vivar & Segundo & \multicolumn{2}{|l|}{ Exdirector del Servicio de Agua Potable de Lima } \\
\hline Jorge Barco Martínez & Segundo & \multicolumn{2}{|c|}{ Exfuncionario del Servicio de Agua Potable de Lima } \\
\hline Miguel Facundo Chinguel & Segundo & \multicolumn{2}{|c|}{ Expresidente de la Comisión de Gracias Presidenciales. } \\
\hline Hammer Zavaleta Gutiérrez & Segundo & \multicolumn{2}{|l|}{ Exdirector del programa "Agua para Todos" } \\
\hline Umberto Olcese Ugarte & Segundo & \multicolumn{2}{|l|}{ Exfuncionario del Servicio e Agua Potable de Lima. } \\
\hline Julio Herrera Pumayauli & Segundo & \multicolumn{2}{|l|}{ Excongresista aprista } \\
\hline
\end{tabular}

Fuente: Ministerio Público. Lima. Perú. 
- Supervisión General.

d. Cuatro principios fundamentales de aplicación obligatoria:

- Descentralización operativa.

- Transparencia institucional.

- Cooperación y

- Coordinación.

Los especialistas coinciden que no sólo hay que reconstruir, sino reordenar el país; en resumen, el FEN según la Cámara de Comercio de Lima (2017), en cifras significó que:

- Hay más de 100000 damnificados

- $\quad$ Se han registrado 242 puentes destruidos

- $\quad$ Se ha perdido alrededor de 198000 viviendas

- A causa del El Niño Costero, el PBI del primer trimestre 2018 crecería apenas $2 \%$.

- Las pérdidas en agro y ganadería superan los 2100 millones de soles (unos 637 millones de dólares)

- El gobierno viene ejecutando 600 millones de soles en la atención de emergencias. (p. 11).

Según anuncio del jefe del INEI (2017): “Cinco sectores económicos registraron cifras negativas en febrero 2017, estos son el sector construcción $(-6,89)$, manufactura $(-3,03)$, electricidad, gas y agua $(-2,28)$, financiero y seguros $(-1,23)$ y agropecuario $(-0,21) ”($ p. 1$)$.

A continuación, el análisis estructural de inversiones de la industria cementera (ver tabla 4).
Su estructura de inversiones explica $85 \%$ en activos corrientes en el 2017 y $88 \%$ en el año previo, su proyección a largo plazo está asegurado porque hubo 177 millones de soles aportado con fondo de accionistas, se fortalece el capital propio que va de $45,4 \%$ a $48,61 \%$, generado por un mejor ingreso de diferencia de cambio por más de 100 millones de soles; gran eficiencia en administración financiera, pero existe riesgo de liquidez a la vista, porque el capital ajeno es superior al capital propio en $2,78 \%$ en términos relativos o 244 millones de soles en términos absolutos.

Otro detalle es la relación de las ventas y su margen, mientras las ventas se incrementaron en $1,8 \%$ en el año 2017, respecto del 2016, las utilidades brutas se redujeron en $2,6 \%$ porque su utilidad bruta operativa se contrajo de $43,80 \%$ a $41,2 \%$. Al final del año 2017 , la empresa tuvo una leve mejora en su competitividad, pero no autonomía en la toma de decisiones pues la composición del capital propio es inferior que el ajeno, no sólo en términos relativos sino también en términos absolutos, pese al incremento de su ganancia neta de $25,6 \%$ el 2016, a $30,2 \%$, el 2017 (ver tabla 5).

Sus inversiones están orientadas al largo plazo, 82\% el 2017 y $77 \%$ el 2016. La mayor desinversión por 277 millones de soles, contribuyó a reducir el capital ajeno en 50 millones y el capital propio con 227 millones de soles, pero no se logró incrementar el capital propio en términos relativos porque este se debilitó de 59,31\% a 57,14\%; sin embargo, las ventas solo cayeron en ese periodo en 15 millones de soles, se destruyó, entre enero-marzo de 2017, toda la infraestructura física de la región norte por efecto climático del Niño Costero, traducido en intensas lluvias en zonas desérticas no preparadas para este fenómeno, una decisión financiera eficiente frente a situaciones fuera de control de la gerencia.

Tabla 4

Análisis Estructural de inversión y financiamiento Unión Andina de Cemento S.A.A. UNACEM (en miles de soles).

\begin{tabular}{|c|c|c|c|c|c|c|}
\hline \multirow{2}{*}{ Conceptos } & \multicolumn{2}{|c|}{2017} & \multicolumn{2}{|c|}{2016} & \multicolumn{2}{|c|}{ Variación } \\
\hline & Valores & $(\%)$ & Valores & $(\%)$ & Valores & (\%) \\
\hline \multicolumn{7}{|c|}{ Inversiones } \\
\hline Activo Corriente & 1349567 & 15,41 & 1068877 & 12,46 & 280690 & 157,90 \\
\hline Activo no Corriente & 7409442 & 84,59 & 7512371 & 87,54 & -102929 & $-57,90$ \\
\hline Total & 8759009 & 100,00 & 8581248 & 100,00 & 177761 & 100,00 \\
\hline \multicolumn{7}{|c|}{ Financiamiento } \\
\hline Capital Ajeno & 4501527 & 51,39 & 4685095 & 54,60 & -183568 & $-103,27$ \\
\hline Capital Propio & 4257482 & 48,61 & 3896153 & 45,40 & 361329 & 203,27 \\
\hline Total & 8759009 & 100,00 & 8581248 & 100,00 & 177761 & 100,00 \\
\hline
\end{tabular}

Fuente: BVL. Estados Financieros Comparados al 30 de setiembre de 2017. 
En cuanto a las ventas y su margen, estas disminuyeron por las razones explicadas en el párrafo anterior en más de 15 millones, 2,5\% inferior al año anterior, pero el margen bruto fue similar al año previo. Al término del año 2017, la empresa ha desmejorado ligeramente en competitividad porque tiene menor autonomía en la toma de decisiones, pues su capital propio creció en menor proporción que el ajeno; además de haber disminuido su ganancia neta de 17,22\% el 2016, a 13,81\%, el 2017, es decir, cayó de 105 millones, en el 2016, a 82 millones de soles en el 2017, en términos absolutos (ver tabla 6).

La estructura de inversiones nos muestra una empresa típicamente industrial que prioriza el largo plazo en lugar del corto plazo: $85 \%$ el 2017 y $88 \%$ el 2016.

La mayor inversión por más de 84 millones de soles se financió con $12 \%$ de capital ajeno y $88 \%$ de capital propio, mejoró su posición del $45,22 \%$ al $46,3 \%$; cuyo efecto notable explica un ahorro en intereses por más 13 millones y otros ingresos de subsidiarias por más de 26 millones de soles. Es una eficiencia en el manejo financiero. Pero existe riesgo de liquidez a la vista porque el capital ajeno es superior al capital propio en $7,4 \%$ en términos relativos o 245 millones de soles en términos absolutos. Otro detalle es la relación de las ventas y su margen, mientras las ventas disminuyeron en $3,94 \%$ en el año 2017, respecto del año previo, las utilidades brutas se redujeron en $1,6 \%$ porque su margen bruta operativa se redujo de $54,08 \%$ a $52,48 \%$. Al final del año 2017, desmejoró su competencia porque no tiene autonomía en la toma de decisiones pues el capital propio es menor que el ajeno, no sólo en términos relativos sino también en términos absolutos, además de haber incrementado su ganancia neta de $21,54 \%$ el 2016, a $22,48 \%$, el 2017.

Mayor rentabilidad patrimonial, tanto en el 2016 y 2017, lo tiene Cementos Yura; mejor uso de capacidad instalada, UNACEM en el 2017 con 4,85\% y Cementos Yura en el 2016, con $4,84 \%$; mejor margen sobre ventas, UNACEM, tanto en el 2017 y el 2016 con $30,13 \%$ y $25,57 \%$, respectivamente.

Tabla 5

Análisis estructural de inversiones y financiamiento cementos Pacasmayo S.A.A. (en miles de soles).

\begin{tabular}{|c|c|c|c|c|c|c|}
\hline \multirow{2}{*}{ Concepto } & \multicolumn{2}{|c|}{2017} & \multicolumn{2}{|c|}{2016} & \multicolumn{2}{|c|}{ Variación } \\
\hline & Valores & $(\%)$ & Valores & $(\%)$ & Valores & (\%) \\
\hline \multicolumn{7}{|c|}{ Inversiones } \\
\hline Activo Corriente & 519823 & 18,10 & 708618 & 22,51 & -188795 & $-68,26$ \\
\hline Activo no Corriente & 2352016 & 81,90 & 2439785 & 77,49 & -87769 & $-31,74$ \\
\hline Total & 2871839 & 100,00 & 3148403 & 100,00 & -276564 & 100,00 \\
\hline \multicolumn{7}{|c|}{ Financiamiento } \\
\hline Capital Ajeno & 1231005 & 42,86 & 1280963 & 40,69 & -49958 & $-18,06$ \\
\hline Capital Propio & 1640834 & 57,14 & 1867440 & 59,31 & -226606 & $-81,94$ \\
\hline Total & 2871839 & 100,00 & 3148403 & 100,00 & -276564 & 100,00 \\
\hline
\end{tabular}

Fuente: BVL. Años 2017-2016 al 30 de setiembre de 2017.

Tabla 6

Análisis estructural de inversión y financiamiento Cementos Yura S.A.A. (en miles de soles).

\begin{tabular}{|c|c|c|c|c|c|c|}
\hline \multirow[t]{2}{*}{ Concepto } & \multicolumn{2}{|c|}{2017} & \multicolumn{2}{|c|}{2016} & \multicolumn{2}{|c|}{ Variación } \\
\hline & Valores & $(\%)$ & Valores & $(\%)$ & Valores & $(\%)$ \\
\hline \multicolumn{7}{|c|}{ Inversiones } \\
\hline Activo Corriente & 512034 & 15,46 & 381474 & 11,82 & 130560 & 154,21 \\
\hline Activo no Corriente & 2799782 & 84,54 & 2845676 & 88,18 & -45894 & $-54,21$ \\
\hline TOTAL & 3311816 & 100,00 & 3227150 & 100,00 & 84666 & 100,00 \\
\hline \multicolumn{7}{|c|}{ Financiamiento } \\
\hline Capital Ajeno & 1778532 & 53,70 & 1767980 & 54,78 & 10552 & 12,46 \\
\hline Capital Propio & 1533284 & 46,30 & 1459170 & 45,22 & 74114 & 87,54 \\
\hline TOTAL & 3311816 & 100,00 & 3227150 & 100,00 & 84666 & 100,00 \\
\hline
\end{tabular}

Fuente: BVL. Años 2017-2016 al 30 de setiembre de 2017. 
Tabla 7

Costo de capital de empresas cementeras año 2016 (en miles de soles).

\begin{tabular}{lrrrrrr}
\hline \multicolumn{1}{c}{ Empresas } & $\begin{array}{c}\text { Activo } \\
\text { Corriente }\end{array}$ & $\begin{array}{c}\text { Pasivo } \\
\text { Corriente }\end{array}$ & $\begin{array}{c}\text { Capital } \\
\text { Neto de } \\
\text { Trabajo }\end{array}$ & $\begin{array}{c}\text { Activo } \\
\text { Fijo } \\
\text { Neto }\end{array}$ & $\begin{array}{c}\text { Total } \\
\text { Recursos }\end{array}$ & $\begin{array}{c}\text { Costo } \\
\text { Capital } \\
\mathbf{7 \%}\end{array}$ \\
\hline UNACEM & 1068877 & $(1012903)$ & 55974 & 8581248 & 8637222 & 604606 \\
C. Pacasmayo & 708618 & $(137055)$ & 571563 & 3148403 & 3719966 & 260397 \\
C. Yura & 381474 & $(293810)$ & 87664 & 3227150 & 3314814 & 232037 \\
TOTAL & 2158969 & $(1443768$ & 715201 & 14956801 & 15672002 & 1097040 \\
\hline
\end{tabular}

Modelo tomado de Arrarte (2012, p. 214).

Fuente: BVL. Años 2017-2016 al 30 de setiembre de 2017.

Tabla 8

Costo de capital de empresas cementeras año 2017 (en miles de soles).

\begin{tabular}{lrrrrrr}
\hline Empresas & $\begin{array}{c}\text { Activo } \\
\text { Corriente }\end{array}$ & $\begin{array}{c}\text { Pasivo } \\
\text { Corriente }\end{array}$ & $\begin{array}{c}\text { Capital } \\
\text { Neto de } \\
\text { Trabajo }\end{array}$ & $\begin{array}{c}\text { Activo } \\
\text { Fijo } \\
\text { Neto }\end{array}$ & $\begin{array}{c}\text { Total } \\
\text { Recursos }\end{array}$ & $\begin{array}{c}\text { Costo } \\
\text { Capital } \\
\mathbf{7 \%}\end{array}$ \\
\hline UNACEM & 1349567 & $(1041362)$ & 308205 & 8759009 & 9067214 & 634705 \\
C Pacasmayo & 519823 & $(122758)$ & 397065 & 2871839 & 3268904 & 228823 \\
C. Yura & 512034 & $(172527)$ & 339507 & 3311816 & 3651323 & 255593 \\
TOTAL & 2381424 & 1336647 & 1044777 & 14942664 & 15987441 & 1119121 \\
\hline
\end{tabular}

Fuente: BVL. Años 2017-2016 al 30 de setiembre de 2017.

Tabla 9

Cálculo del valor económico agregado del año 2016.

\begin{tabular}{lcccccc}
\hline \multicolumn{1}{c}{ Empresas } & U.O.A.I & I. R. 27\% & U.N.D.I. & C. D. C. & E. V. A. & (\%) \\
\hline UNACEM & 624651 & $(168656)$ & 455995 & $(604606)$ & $(148611)$ & 49,83 \\
C Pacasmayo & 156238 & $(42184)$ & 114054 & $(260397)$ & $(146343)$ & 49,07 \\
C. Yura & 313383 & $(84613)$ & 228770 & $(232037)$ & $(3267)$ & 1,10 \\
TOTAL & 1094272 & $(295453)$ & 798819 & 1097040 & $(298221)$ & 100,00 \\
\hline
\end{tabular}

U.O.A.I. = Utilidad Operativa Antes de Impuestos.

I. $\boldsymbol{R} .=$ Impuesto a la Renta. $27 \%$.

U.N.D.I. = Utilidad Neta después de Impuestos.

C.D.C. $=$ Costo de Capital

E V. A. = Valor Económico Agregado.

Fuente: BVL. Años 2017-2016 al 30 de setiembre de 2017.

Tabla 10

Cálculo del valor económico agregado del año 2017.

\begin{tabular}{lccccrr}
\hline \multicolumn{1}{c}{ Empresas } & U.O.A.I. & I.R. $\mathbf{2 7} \%$ & U.N.D.I. & C.D.C. & \multicolumn{1}{c}{ E.V.A. } & \multicolumn{1}{c}{ (\%) } \\
\hline UNACEM & 604529 & $(163223)$ & 441306 & $(634705)$ & $(193399)$ & 51,52 \\
C Pacasmayo & 138511 & $(37398)$ & 101113 & $(228823)$ & $(127710)$ & 34,02 \\
C. Yura & 275762 & $(74456)$ & 201306 & $(255593)$ & $(54287)$ & 14,46 \\
TOTAL & 1018802 & $(275077)$ & 743725 & $(1119121)$ & $(375396)$ & 100,00 \\
\hline
\end{tabular}

U.O.A.I. = Utilidad Operativa Antes de Impuestos. I. R. = Impuesto a la Renta

U.N.D.I. = Utilidad Neta después de Impuestos. $\quad$ C.D.C. $=$ Costo de Capital

E V. A. = Valor Económico Agregado.

Fuente: BVL. Años 2017-2016 al 30 de setiembre de 2017. 


\section{DISCUSIÓN}

Como puede notarse, el modelo da para muchas variaciones, Bonilla (2010) sostiene que a la utilidad después de impuestos debe agregarse los gastos financieros, restar las utilidades extraordinarias y agregarse las pérdidas extraordinarios, finalmente, a este resultado, deducir el costo financiero de los recursos totales. Posición con la que estamos en total descuerdo porque los ejercicios económicos anuales pueden ser prósperos como negativos, sobre todo en tiempos como este, en el que el cambio climático puede modificar el panorama empresarial como el FEN Costero, motivo de esta investigación, porque en adelante nuestras empresas ubicadas en determinadas zonas geográficas del territorio nacional, ya estarán tomando medidas preventivas, como seguros contra accidentes climáticos para minimizar las futuras pérdidas que pudieran generarse. Nuestros cálculos se basaron en las ideas de Izar (2007) y en la experiencia profesional adquirida en el sector privado nacional:

$\mathrm{EVA}=\mathrm{UODI}-\mathrm{k}_{0}$ (Recursos).

Donde:

EVA = Valor Económico Agregado, \$

UODI = Utilidad después de Impuestos, \$

$\mathrm{k}_{0}=$ Costo de Capital, \%

Sustentada en dos consideraciones claves que respaldan la importancia del modelo que proponemos, la tasa de interés aplicado y su práctica continua y uniforme basada en el principio de consistencia. Es importante conocer estos temas, ya que no se publican oficialmente en el Perú, son estudios privados que manejan los Directorios pero que no alcanzan a la opinión pública. En materia de corrupción no está demostrado la participación de los productores de la industria cementera peruana, mientras que el FEN, sí tuvo gran impacto en la infraestructura física de las regiones de La Libertad, Lambayeque, Piura y Tumbes. Se generó una caída en ventas, utilidades y el valor económico agregado (EVA) en 2016-2017 en la empresa de Cementos Pacasmayo, además de daños materiales a su planta industrial, por estar ubicada al norte del país, lugar donde ocurrió este fenómeno.

\section{REFERENCIAS BIBLIOGRÁFICAS}

Agencia de los Estados Unidos para el Desarrollo Internacional. (1999). Declaración de principios. New York: Casals \& Associates. Inc.
Arrarte, R. (2012) ¿Es competitiva la industria cementera peruana? Contaduría Universidad de Antioquia, 61, 195-218.

Banco Mundial (2000). Declaración de principios. New York: Ediciones Mundi-Prensa.

Bolsa de Valores de Lima. (2016). Estados Financieros Comparados. Lima. Recuperado de: https://www.bvl. com.pe/pubdif/eeffcom/ef201612.pdf

Bolsa de Valores de Lima. (2017). Estados Financieros Comparados. Lima. Recuperado de: https://www.bvl. com.pe/pubdif/eeffcom/ef201712.pdf

Bonilla, F. (2010). El valor económico agregado (EVA) en el valor del negocio. Revista Nacional de Administración, 1 (1): 55-70

Cámara de Comercio de Lima (2017). No sólo hay que reconstruir, sino reordenar el país (Informe Especial). La Cámara, 771, 10-13. Recuperado de: https://www.camaralima.org.pe/repositorioaps/0/0/par/edicion771/ ed_dig_771.pdf

Congreso de la República. (2018). Informe Final de Comisión Lava Jato. Lima. Recuperado de: http://www. congreso.gob.pe/index.php?K=263\&id $=11818 /$ noticias/LAVA-JATO-DESTAPAR\%C3\%81-A-CORRUPTOS-EN-INFORME-FINAL\#.XUjq8fJKiM8

Contreras, A., Martínez, F., Regalado, F. \& Vásquez, K. (2017). Impacto del Fenómeno de El Niño a la economía peruana. Lima: Asociación Peruana de Economía.

Instituto Nacional de Estadística y Censo INEI (2017). Comportamiento de la economía peruana en el cuarto trimestre de 2016 (Informe No. 1). Lima.

Instituto Nacional de Estadística y Censo INEI (2017). Producción nacional creció en $0,74 \%$ en febrero 2017 y acumuló 91 meses de crecimiento consecutivo (Nota de Prensa). Recuperado de: https:// www.inei.gob.pe/prensa/noticias/produccion-nacional-crecio-en-074-en-febrero-2017-y-acumulo-91-meses-de-crecimiento-consecutivo-9675/

Instituto Nacional de Estadística y Censo INEI (2018). Comportamiento de la economía peruana en el cuarto trimestre de 2017 (Informe No. 1). Lima.

Izar, J. (2007). El modelo del valor económico agregado (EVA). Contabilidad Gerencial. México D.F: Trillas.

Ministerio Público (2018). Cuadro de involucrados de acuerdo a la Fiscalía. Lima. 
Quiroz, A. (2013). Historia de la corrupción en el Perú. Lima, IEP; Instituto de Defensa Legal, (Perú Problema, 38). Virginia: Casals \& Associates, Inc.

USAID para América Latina (2004). La Corrupción en América Latina: Estudio Analítico Basado en una Revisión Bibliográfica. Casals \& Associates. Inc.
Volosin, N. (2015). Datos abiertos, corrupción y compras públicas. Santiago de Chile: (ILDA). Iniciativa Latinoamericana por los Datos Abiertos.

Weston, J. (1995) Finanzas en Administración. México DF: McGraw-Hill 